\title{
Advanced Medical Simulation: Interactive Videos and Rapid Cycle Deliberate Practice to Enhance Teamwork and Event Management
}

\section{Effective Event Management during Simulated Obstetrical Cases}

\author{
https://doi.org/10.3991/ijac.v12i3.11270 \\ Fernando Salvetti $\left({ }^{凶}\right)$ \\ Centro Studi Logos, Turin, Italy \\ Logosnet, Lugano, Switzerland and Houston, Texas, USA \\ salvetti@logosnet.org \\ Roxane Gardner \\ Center for Medical Simulation, Massachusetts, USA \\ Brigham and Women's Hospital / Children's Hospital / Massachusetts General Hospital and \\ Harvard Medical School, Massachusetts, USA \\ Rebecca Minehart \\ Massachusetts General Hospital and Harvard Medical School, Massachusetts, USA \\ Center for Medical Simulation, Massachusetts, USA \\ Barbara Bertagni \\ Centro Studi Logos, Turin, Italy \\ Logosnet, Lugano, Switzerland and Houston, Texas, USA
}

\begin{abstract}
This article is about an experiential coursework focused on learning and improving teamwork and event management during simulated obstetrical cases. Effective team management during a crisis is a core element of expert practice. Medical simulation can contribute enormously to enhance teamwork during a crisis, fostering situational awareness and contextual intelligence, as well as cognitive retention of essential steps and procedures to be performed during an ongoing crisis. A crisis management organizational approach known as Name-Claim-Aim is being used to facilitate crisis management and decision making. Augmented and virtual reality technology (e-REAL) reproduces a variety of different emergent situations, enabling learners to interact with multimedia scenarios and practice using Name-Claim-Aim in a virtual environment. Subsequently, learners rapidly cycle between deliberate practice and directed feedback within a high-fidelity simulation scenario until mastery is achieved. So far, the early findings show that visualization, if linked in interactive ways to the learners, allows for the better use of the neural processes and for better learning and meaningful behavior change. Non-invasive technologies to monitor patterns of neural activity during the program delivery will be introduced.
\end{abstract}


Keywords-Teamwork, interactive visualization, contextual intelligence, cognitive retention, advanced medical simulation

\section{Introduction}

Teamwork and Crisis Resource Management for Labor and Delivery Clinicians (Introductory and Advanced Levels) is experiential coursework focused on learning and improving teamwork and event management during simulated obstetrical cases. It's an interprofessional program based on advanced simulation, delivered many times per year in Boston, Massachusetts, at the Center for Medical Simulation (CMS) in a realistic clinical setting, equipped with all the supplies and devices normally found in Labor and Delivery. Each case is immediately followed by a facilitated debriefing led by experienced instructors and faculty members of CMS. Participants include obstetricians, obstetrical nurses, midwives and obstetrical anesthesiologists, since collaboration and teamwork among these groups is so important during obstetrical events [1].

\section{How to Best Organize a Labor and Delivery Team in a Crisis}

Effective team management during a crisis is a core element of expert practice. Medical simulation can contribute enormously to enhance teamwork during a crisis, fostering situational awareness and contextual intelligence, as well as cognitive retention of essential steps and procedures to be performed during an ongoing crisis.

A crisis management organizational approach using a mnemonic called NameClaim-Aim is being used in order to facilitate crisis management and decision making: medical knowledge and skills are essential components of the decisions and actions performed during crises, but they are not enough since what has to be managed is the entire situation - including the environment, the equipment and the patient care team.

After decades' worth of dedicated simulation education for anesthesiologists and labor and delivery teams, teamwork experts at the CMS have found that these teams still struggle to routinely organize in crises during simulation courses, let alone in the clinical environment. Stories from course participants of all professions uphold this notion that there exists a real challenge to both focus on the clinical picture and apply organization principles to the team and, more often than not, the organization is under-prioritized. Part of this may be due to the intense cognitive load experienced by many who are managing a stressful clinical crisis; and it can be difficult to also remember the 11-crisis resource management (CRM) principles introduced by Gaba, Fish and Howard [2] and apply them routinely while actively managing a resuscitation. 


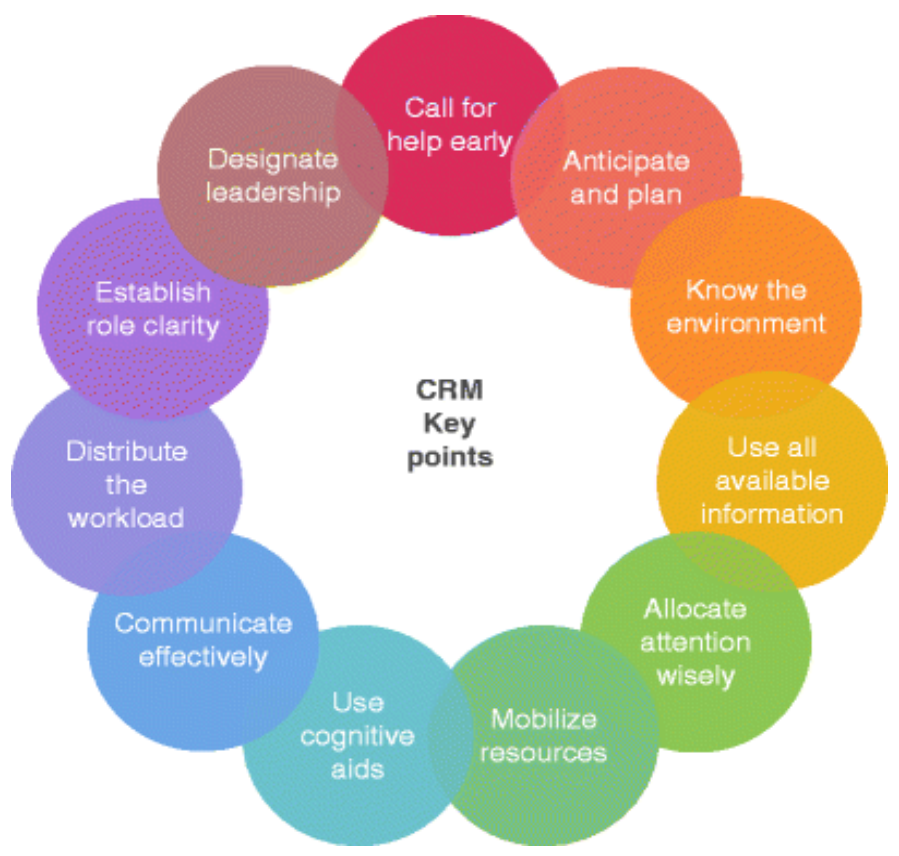

Fig. 1. Crisis Resource Management - Key Points

Appreciating the impact of stress on high level thinking [3], faculty at CMS collapsed these 11 key points into five key CRM concepts of role clarity, effective communication, effective use of personnel, effective management of resources and global assessment - see Figure 2.

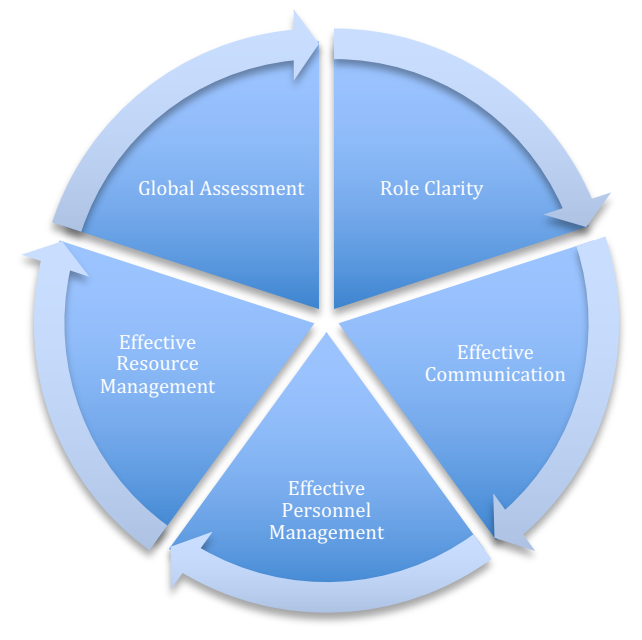

Fig. 2. CMS - Five Key CRM Concepts 
The role of "Event Manager," rather than "Team Leader," is expressly promoted at CMS to facilitate distributed leadership in crises, which has proven to be effective in teams of expert practitioners, and which deliberately seeks to collapse hierarchies which may inhibit speaking-up behavior from team members [4] with the goal of avoiding inattentional blindness and failure of perception. The role of "Event Manager" is one who coordinates the communication and efforts of the team and oversees the organization and application of CRM principles in addition to actively soliciting input and decision-making regarding medical care, if necessary. As role clarity repeatedly is a problem for teams, often manifesting in multiple providers being unsure how to help while critical roles are duplicated or left unfilled, and the "Event Manager" acts to facilitate role designation, orchestrate and coordinate team function. Remembering roles can be challenging as well during a crisis due to high cognitive load, an unnecessary problem for teams.

Based on these challenges, even with a 5 Key CRM concept framework, the mnemonic "Name-Claim-Aim" was developed at CMS to incorporate 10 of the 11 CRM principles in an easy-to-remember, and easily applied, framework - see Figure 3. Cognitive aids were developed to help facilitate learning of this mnemonic and an "Event Manager Checklist" was created to facilitate designating roles effectively see Figure 4 and Figure 5. Participants have been given this cognitive aid, designed as an ID badge-sized card, to easily access during their simulation course. In addition, the "Name-Claim-Aim" and "Event Manager Checklist" have been adopted by the Massachusetts General Hospital (MGH) (Boston, MA, USA) for inclusion on the latest version of their Emergency Manuals - see Figure 6, which are distributed throughout all anesthetizing locations and offsite healthcare facilities affiliated with MGH.

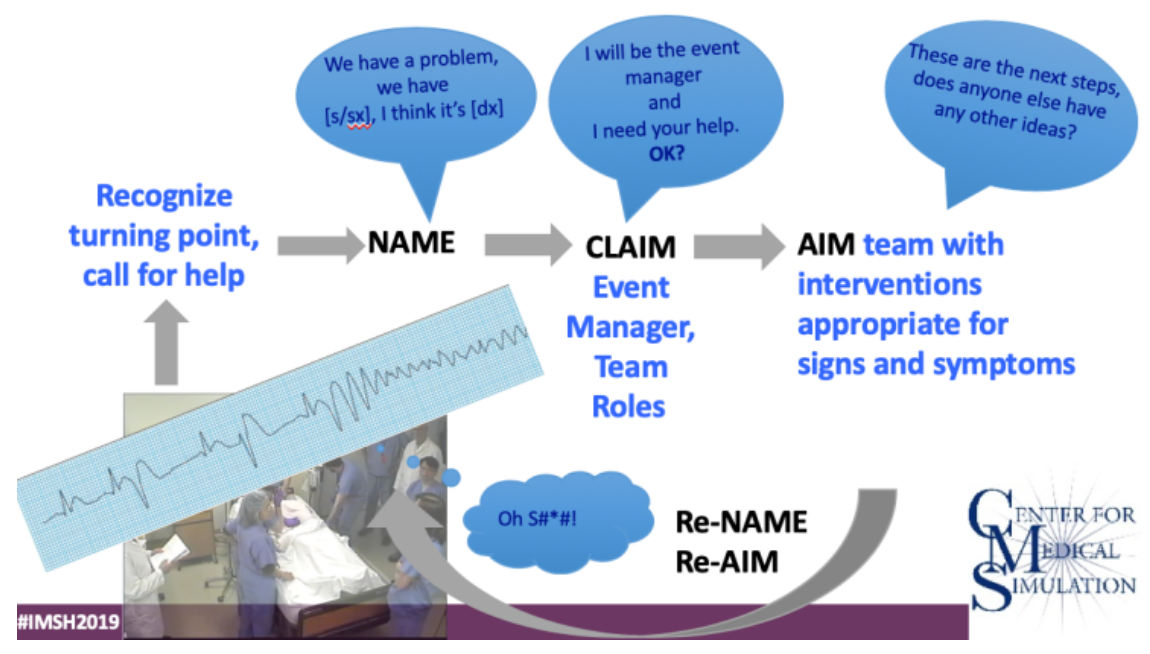

Fig. 3. Application of Name-Claim-Aim 


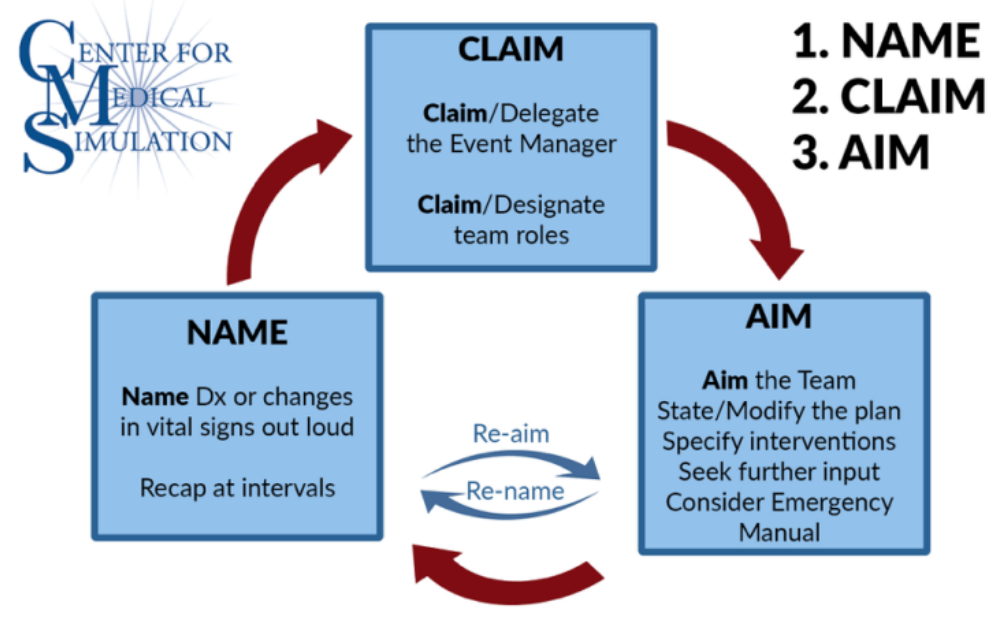

Fig. 4. Name-Claim-Aim Cognitive Aid

\section{BASIC Roles}

1. Event Manager

2. Circulation -- CPR

3. Airway

4. Drugs

5. Recorder / Timer

6. Resource Person

\section{Additional Roles}

IVs, Fluids, Labs

Hx \& Chart Review

Liaison w/ External Services \& Phone

Security \& Crowd Control

Technical Equipment

Monitor Watcher

Emergency Manual Reader

Fig. 5. Name-Claim-Aim Cognitive Aid 


\section{MANAGING CRITICAL EVENTS}

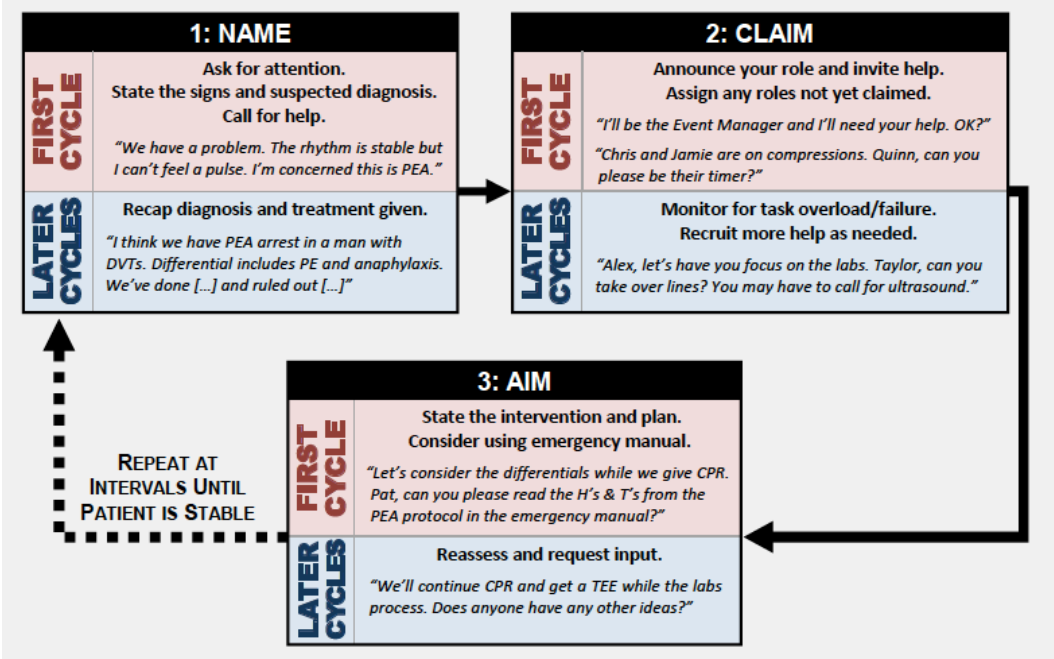

Fig. 6. Massachusetts General Hospital (MGH) (Boston, MA, USA) inclusion of Name-ClaimAim in the latest version of their Emergency Manuals

\section{Interactive Videos and Rapid Cycle Deliberate Practice}

Rapid Cycle Deliberate Practice (RCDP) is a novel simulation-based education model that is currently attracting interest, implementation, exploration and research in medical education. In RCDP, learners rapidly cycle between deliberate practice and directed feedback within the simulation scenario until mastery is achieved [5]. Common RCDP implementation strategies include: splitting simulation cases into segments, micro-debriefing in the form of "pause, debrief, rewind and try again" and providing progressively more challenging scenarios. During the Labor \& Delivery program, clinicians are shown short dynamic videos: they are challenged to recognize a situation requiring rapid intervention, communication, knowledge sharing, decisionmaking and management of unforeseen events - taking into consideration critical contextual factors such as a lack of time, scarcity of resources and tools, and a multitude of impactful factors. Augmented and virtual reality technology is being used (eREAL), enabling learners to interact with multimedia scenarios reproducing very different situations [6]. Learners are requested to be compliant with the Name-ClaimAim mnemonic in order to manage the crisis by coordinating the team roles and efforts. The interactive videos feature unexpected clinical or non-clinical, emergent scenarios, including extreme, dangerous environmental threats - see figures 7-9.

In 2018, 51 experienced labor and delivery clinicians participated in RCDP within the e-REAL virtual environment, using Name-Claim-Aim for organizing a team to manage the crisis. These learners were highly positive about their experience. Some commented that they "loved the video rehearsals", "will practice the Event Manager 
role", "will use Name-Claim-Aim in clinical practice", and "will apply the strategy of Name-Claim-Aim and establish role of the Event Manager". A few learners became so activated by the interactive videos that they performed CPR on an imagined person collapsed on the floor despite the absence of actual mannequins or other real equipment.

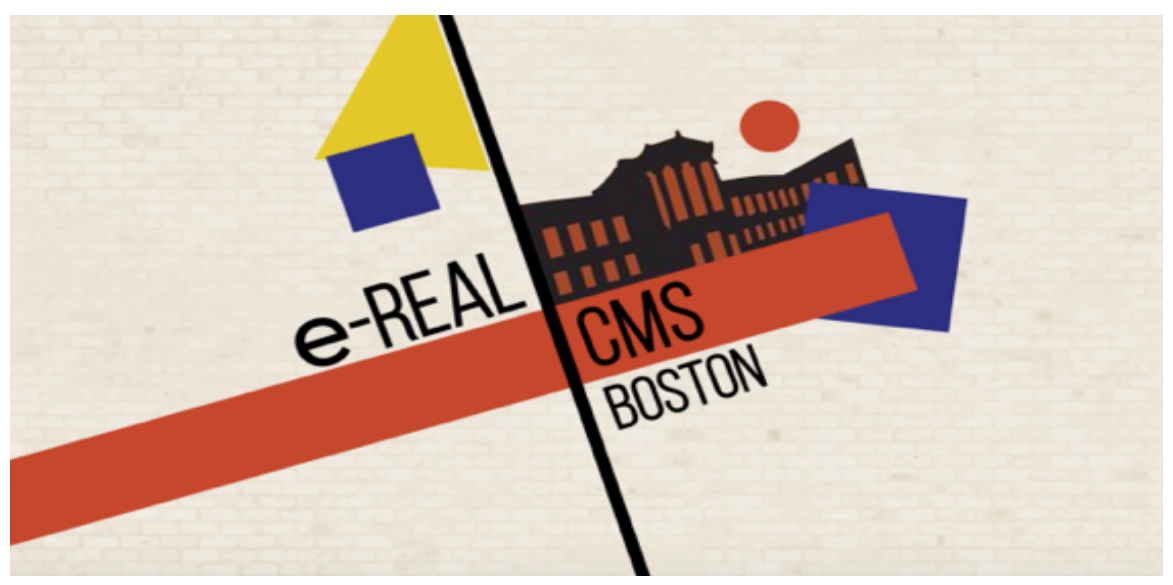

Fig. 7. The wall ready to operate as an interactive screen

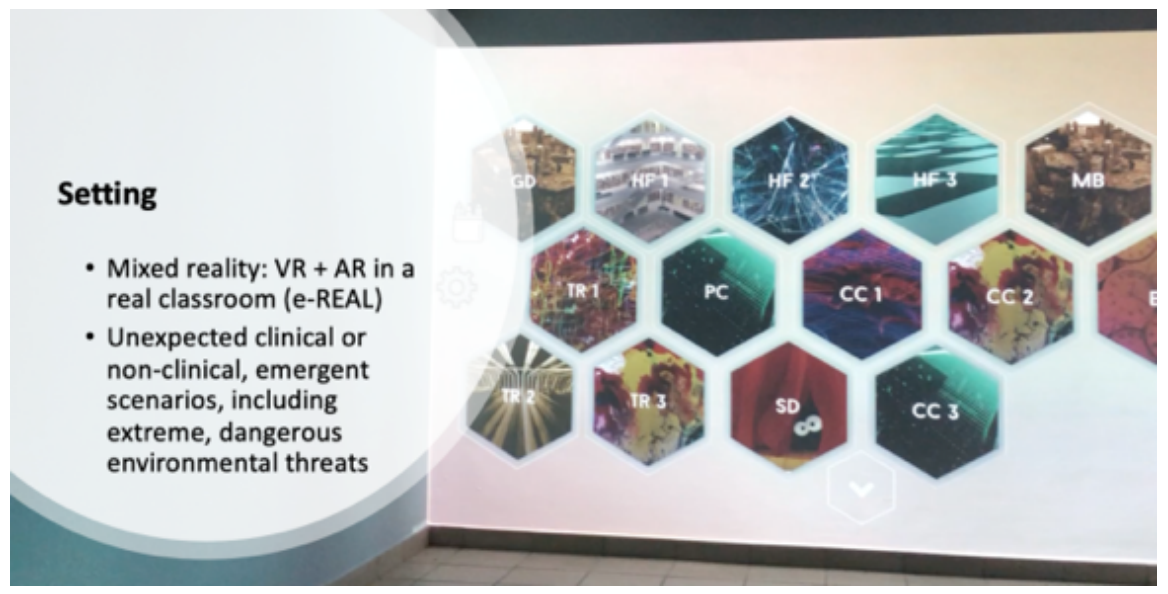

Fig. 8. Videos' menu within a mixed reality setting 


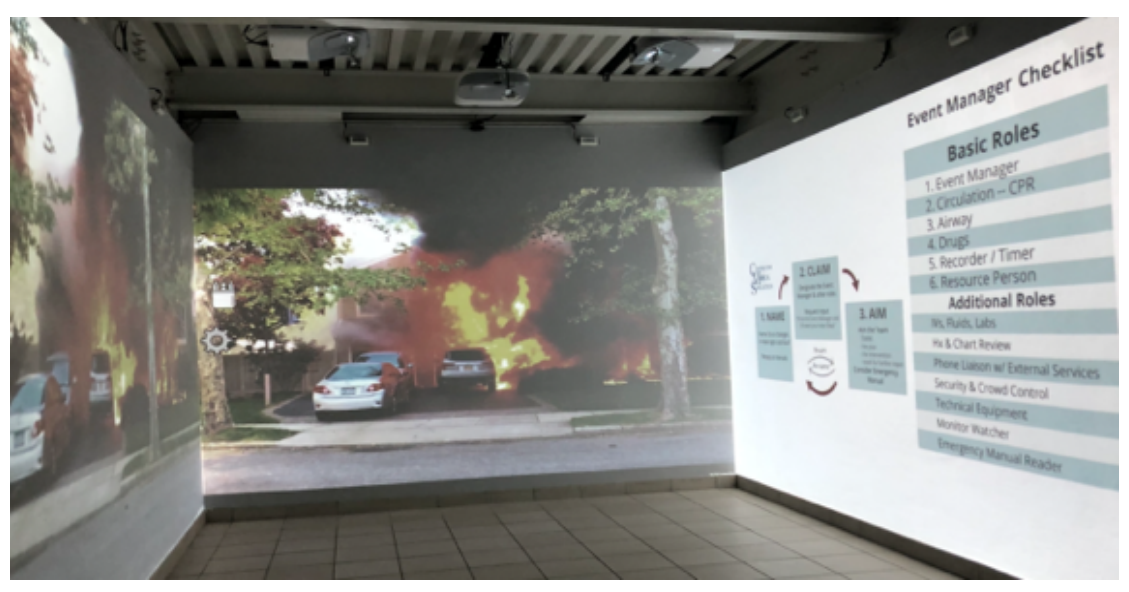

Fig. 9. Sample content: Home Fire Emergency

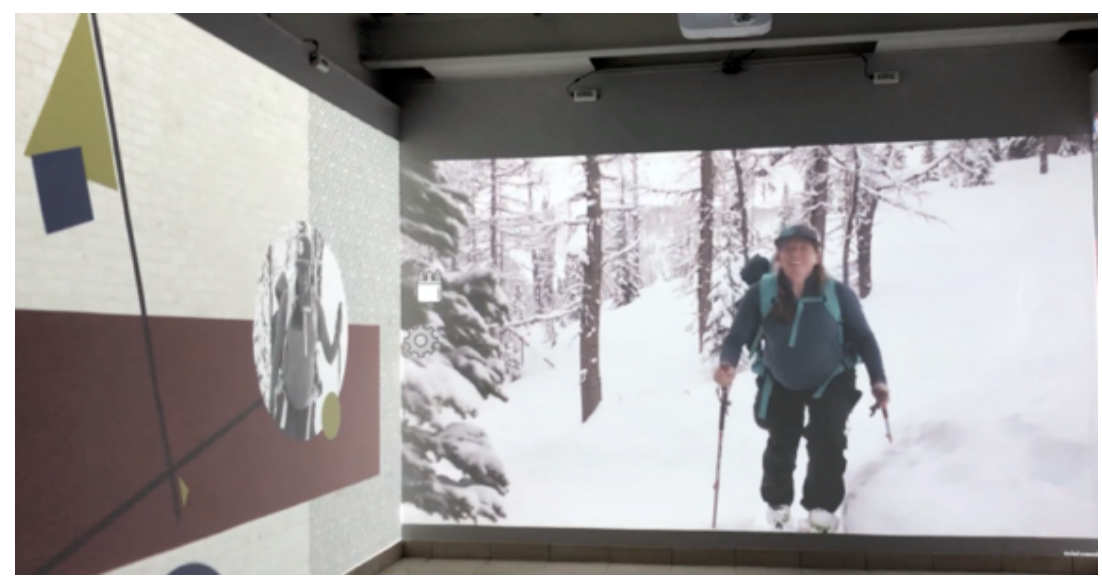

Fig. 10. Sample content: Clinical Emergency

\section{Visual Storytelling, Effective Learning and Open Questions}

The learning setting is an e-REAL system that helps create interactive virtual and augmented reality environments for advanced simulation. It is a system that allows a team to be immersed together in a mixed reality environment where they can interact with virtual projections in a natural way, that doesn't require them to wear headsets to be seen. The e-REAL technology, thanks to special projectors and touch-tracking cameras, turns simple walls and empty spaces into interactive environments controlled by the touch of the hand [7].

e-REAL is the output of an applied research project, still ongoing, started and supported by Logosnet in 2011, developed in collaboration with top research and simulation centers: CMS in Boston, Environmental Design and Multisensorial Experience Lab (EDME) from the Polytechnic School of Milan, SIMNOVA Medical Simulation 
Center from the University of Eastern Piedmont in Novara, Department for Automation and Informatics (DAUIN) from the Polytechnic School of Turin, Training Center of the Red Cross of Italy in Bologna (CRIBO), National Research Council of Italy (CNR) and others.

The learning setting is an e-REAL system that helps create interactive virtual and augmented reality environments for advanced simulation. It is a system that allows a team to be immersed together in a mixed reality environment where they can interacwith virtual projections in a natural way, that doesn't require them to wear headsets to be seen. The e-REAL technology, thanks to special projectors and touch-tracking cameras, turns simple walls and empty spaces into interactive environments controlled by the touch of the hand [7].

e-REAL is the output of an applied research project, still ongoing, started and supported by Logosnet in 2011, developed in collaboration with top research and simulation centers: CMS in Boston, Environmental Design and Multisensorial Experience Lab (EDME) from the Polytechnic School of Milan, SIMNOVA Medical Simulation Center from the University of Eastern Piedmont in Novara, Department for Automation and Informatics (DAUIN) from the Polytechnic School of Turin, Training Center of the Red Cross of Italy in Bologna (CRIBO), National Research Council of Italy (CNR) and others.

In the e-REAL environment, learners are immersed in different scenarios designed by visual storytelling techniques that allow them to practice handling realistic situations, rather than learning facts or techniques out of context. Much like being immersed within a videogame, people are challenged by facing real cases within complex scenarios that present a more than real wealth of information.

The system enables a multilayer vision: the many levels of the situation are made available simultaneously, by overlaying multisource info-e.g. words, numbers, images, etc. - as within an augmented reality display, but without the need to wear special glasses. Visualizations within e-REAL show relationships between topics, activate involvement, generate questions that learners didn't think of before and facilitate memory retention. So visualizations act like concept maps to help organize and represent knowledge on a subject in an effective way.
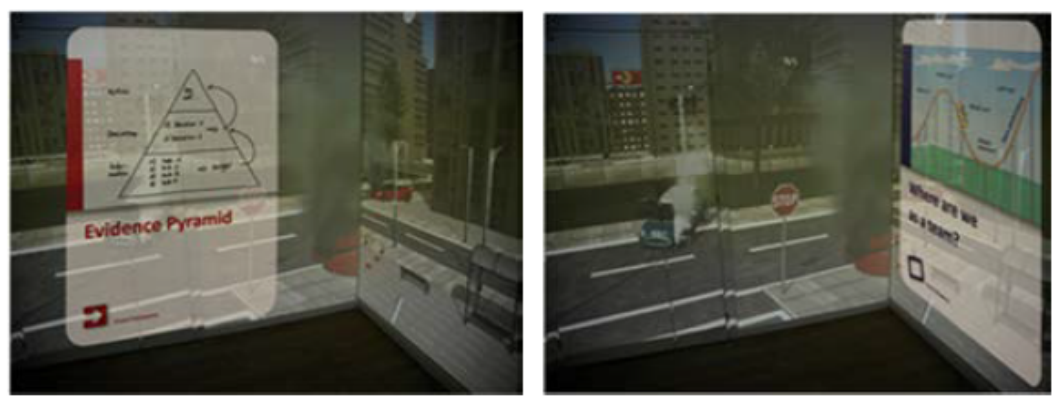

Fig. 11.Multilayer visualization 
By visualizing relations between topics, contextual factors, cognitive maps and dynamic cognitive aids, e-REAL allows learners for the better use of the neural processes and for storing information into memories based on experiences. At the same time, it helps instructors to immediately identify errors and difficulties of the trainees facilitating an effective debriefing.

The project is ongoing and we are trying to answer two key questions:

- Is visual storytelling enabling more situational awareness, contextual intelligence and cognitive retention translating into a simulated clinical crisis?

- Does the association between visual storytelling and the mnemonic "Name-ClaimAim" facilitate awareness and teamwork in a simulated clinical crisis?

So far, the early findings show that an interactive learning environment is an effective simulation tool for Labor and Delivery because it offers realistic scenarios that can be easily reconfigured to generate many different situations, including extreme and dangerous ones. Brain activity from thoughts, visualization, evocation of memories and emotions contributes to the learning process, mainly during the early stage of a simulation-based program.

\section{More to Explore}

We assume that functional changes in neural circuitry, associated with learning, occur best when the learner is actively engaged. We are currently working to introduce non-invasive technologies to monitor patterns of neural activity during the program delivery because the application of computational and quantitative behavioral approaches combined with functional brain imaging can reveal important information about the strategies employed by human brains to acquire, store, and retrieve information in a variety of tasks and settings.

Further questions we're aimed at answering are the following: How does a special class of brain cells, called mirror neurons, reflect the outside world, revealing a new avenue for human understanding, connecting, and learning? [8]. What happens when we learn a new action pattern? Do mirror neurons play a role in this? If so, may we learn just by mirroring? May the mirror system serve as a bridge that allows us to teach and learn new skills? The simpler the pattern, the easier the task? Can pattern recognition be applied to the most complex and even strange situations? Are emotions contagious? When we see someone laugh, cry, show disgust, or experience pain, are we sharing that emotion?

One of the most exciting events in neurosciences over the past few years has been the discovery of a mechanism that unifies action perception and action execution. The essence of this "mirror" mechanism is as follows: whenever individuals observe an action being done by someone else, a set of neurons that code for that action is activated in the observers' motor system. Since the observers are aware of the outcome of their motor acts, they also understand what the other individual is doing without the need for intermediate cognitive mediation [9]. 
Sets of mirror neurons appear to encode templates for specific actions. This property may allow an individual not only to perform basic motor procedures without thinking about them, but also to comprehend those acts when they are observed-without any need for explicit reasoning about them. Many aspects of imitation have long perplexed neuroscientists, including the basic question of how an individual's brain takes in visual information and translates it to be reproduced in motor terms. If the mirror neuron system serves as a bridge in this process, then in addition to providing an understanding of other people's actions, intentions and emotions, it may have evolved to become an important component in the human capacity for observation-based learning of sophisticated cognitive skills.

We are aimed at reaching a better understanding by performing experiments never done before in medical simulation.

\section{$6 \quad$ References}

[1] https://harvardmedsim.org/course/learning-teamwork-and-crm/

[2] Gaba D.M., Fish K.J, Howard S.K. (1994). Crisis Management in Anesthesiology. Churchill Livinstone, Philadelphia, PA; Walker J.D., Spencer P.J., Walzer T.B., Cooper J.B. (2018). Simulation in Cardiac Surgery, in Cohn L.H., Adams D.H. (Eds.). Cardiac Surgery in Adult, McGraw Hill, Columbus, OH.

[3] Arnsten AF. Catecholamine modulation of prefrontal cortical cognitive function. Trends in cognitive sciences. Nov 1 1998; 2(11): 436-447. https://doi.org/10.1016/s13646613(98)01240-6

[4] Raemer D.B., Kolbe M., Minehart R.D., Rudolph J.W., Pian-Smith M.C. (2016). Improving Anesthesiologists' Ability to Speak Up in the Operating Room: A Randomized Controlled Experiment of a Simulation-Based Intervention and a Qualitative Analysis of Hurdles and Enablers. Acad Med. Apr; 91(4):530-9. https://doi.org/10.109 7/acm.0000000000001033

[5] Taras J., Everett T. (2017). Rapid Cycle Deliberate Practice in Medical Education - a Systematic Review. Cureus Apr. 9(4): e1180. DOI 10.7759/cureus.1180

[6] Salvetti F., Bertagni B. (Eds.) (2018). Learning 4.0. Advanced Simulation, Immersive Experiences and Artificial Intelligence, Flipped Classrooms, Mentoring and Coaching. Franco Angeli, Milan.

[7] https://e-real.net/healthcare/

[8] Rizzolatti G., Sinigaglia C. (2008). Mirrors in The Brain: How Our Minds Share Actions and Emotions. Oxford University Press: Oxford and New York, NY.

[9] Rizzolatti G., Cattaneo G., Fabbri-Destro M. (2009). Mirror Neurons and their Clinical Relevance. Nature. Jan. 9(5): 24-34. https://doi.org/10.1038/ncpneuro0990

\section{$7 \quad$ Authors}

Fernando Salvetti (J.D., P.P.E., M.Phil., Ph.D. - salvetti@logosnet.org), Founder of Logosnet in Turin, Lugano, Berlin and Houston, is an epistemologist, an anthropologist and a lawyer who co-designed e-REAL, the enhanced reality lab where virtual and real worlds are merging within an advanced simulation environment. He is 
committed to exploring virtual and augmented reality, cognitive aids by artificial intelligence, visual thinking, interactive and immersive learning, emerging scenarios and trends, and cross-cultural intelligence. He is also Adjoint Professor of Communication of Science at the University "Bicocca" of Milan.

Rebecca D. Minehart (M.D., M.S.H.P.Ed. - rminehart@mgh.harvard.edu), Director for Anesthesia Clinical Courses at the Center for Medical Simulation in Boston (CMS), is an obstetric anesthesiologist at Massachusetts General Hospital (MGH), an Assistant Professor of Anesthesia at Harvard Medical School, and the Program Director for the MGH Obstetric Anesthesia Fellowship Program. She is an ardent education and patient safety advocate who has been involved in international efforts to both research and promote best teamwork and communication practices, especially involving speaking up and giving feedback. She is a recognized expert in educational techniques utilizing simulation and is a core teaching faculty member at both CMS and the MGH Learning Laboratory, where she serves as the Operating Room Simulation Officer.

Roxane Gardner (M.D., M.H.P.E., M.P.H., Ph.D. - rgardner1@bwh.harvard.edu), Senior Director for Clinical Programs and Director of the Visiting Scholars and Fellowship Program at the Center for Medical Simulation in Boston (CMS), has been a principle faculty member of CMS since 2002 and Co-Director of its Labor and Delivery Teamwork and Crisis Management program since its inception in 2003. In addition to her roles at CMS, Dr. Gardner is an Assistant Professor of Obstetrics, Gynecology and Reproductive Biology at the Harvard Medical School and holds appointments in Boston at Brigham and Women's Hospital, Boston Children's Hospital, and Massachusetts General Hospital.

Barbara Bertagni (B.Sc., B.A., M.A., M.Phil., Ph.D., Clin. Psy.D. bertagni@logosnet.org) - Founder of Logosnet and e-REAL co-designer, is a clinical psychologist, an anthropologist and a practical philosopher particularly involved with personal and professional development, coaching and mentoring, immersive learning and advanced simulation. She works as a sparring partner, a coach and a mentor advising people and organizations across the globe.

This article is a revised version of a paper presented at the International Conference on E-Learning in the Workplace 2019 (ICELW 2019), held in June 2019, at Columbia University in New York, NY, USA. Article submitted 2019-07-13. Resubmitted 2019-08-23. Final acceptance 2019-08-24. Final version published as submitted by the authors. 